\title{
CONCEPÇÃO DE PROFESSORES EM FORMAÇÃO INICIAL: CONHECIMENTO SOBRE A TECNOLOGIA ASSISTIVA DOSVOX
}

\author{
Jamile Santinello \\ (Dhttps://orcid.org/0000-0003-1136-2421 \\ Eliziane de Fátima Alvaristo ${ }^{2}$ \\ (1Dhttps://orcid.org/0000-0002-1549-4176 \\ Lucia Virginia Mamcasz Viginheski ${ }^{3}$ \\ (Dhttps://orcid.org/0000-0002-6474-0927
}

Resumo: 0 uso da Tecnologia Assistiva- TA- tem ampliado a autonomia e possibilitado à inclusão de estudantes com deficiência nos âmbitos educacionais de ensino. Para tanto, é importante os professores qualificarem-se para fazer uso destas tecnologias em salas de aula. Este estudo objetiva investigar a concepção de professores em formação inicial de um curso de Pedagogia sobre o conhecimento da Tecnologia Assistiva - Software Dosvox para educação inclusiva, a projetar o processo de ensino e aprendizagem de estudantes com deficiencia visual. Utilizou-se de abordagem qualitativa de natureza descritiva, tendo como estratégia o estudo de caso. Foi realizado em uma Instituição de ensino Superior Pública, localizada no interior do estado do Paraná, e, traz como participantes vinte e cinco professores em formação inicial. Os instrumentos utilizados foram questionários com questões abertas. Os dados foram analisados a partir da análise de conteúdo, divididos em três categorias a: i) concepção sobre deficiência visual; ii) educação inclusiva; iii) tecnologia assistiva. Os resultados revelam que existem lacunas em relação ao conhecimento dos professores sobre a TA para o processo de ensino e aprendizagem à estudantes com deficiência visual, atentando para inclusão de disciplinas no currículo das licenciaturas, a abordar a aplicação de teorias e práticas consistentes.

Palavras-chave: Tecnologia assistiva. Software Dosvox. Formação de professores inicial. Deficiência visual. Inclusão.

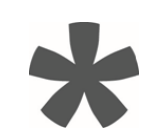

1 Doutora em Comunicação (ECO-UFRJ-2010-2013). Mestre em Educação (UEM/PR-2004-2006). Especialista em Computação aplicada ao Ensino (UEM/PR-1999). Atualmente é Professora efetiva da Universidade Estadual do Centro-Oeste- UNICENTRO, no Departamento de Pedagogia e Mestrado em Educação.

2 Mestre em Ensino de Ciência e Tecnologia (UTFPR). Graduada em Pedagogia (UNICENTRO). Especialização em Educação Especial - (Univale). Atualmente é docente no Centro de Atendimento Especializado na área de deficiência visual - Apadevi. Docente de Sala de Recursos Multifuncionais SEMEC.

3 Graduada em Licenciatura em Matemática pela Universidade Estadual do Centro-Oeste (1996). Mestra em Ensino de Ciência e Tecnologia pela (UTFPR - 2013). Doutora em Ensino de Ciência e Tecnologia pelo Programa de Pós Graduação em Ensino de Ciência e Tecnologia (UTFPR - 2017). Docente do Programa de Pós Graduação em Promoção da Saúde do Centro Universitário UniGuairacá. 


\title{
CONCEPTION OF INITIAL TRAINING TEACHERS: KNOWLEDGE ABOUT DOSVOX ASSISTIVE TECHNOLOGY
}

\begin{abstract}
The use of Assistive Technology has expanded the autonomy and enabled the inclusion of students with disabilities in the educational scope of teaching. Therefore, it is important for teachers to qualify to use these technologies in classroom. This study aims to investigate the conception of teachers in initial formation of a Pedagogy course on the knowledge of Assitive Technology - Dosvox Software for inclusive education, to project the teaching and learning process of visually impaired students. A qualitative approach of a descriptive nature was used, having as a strategy the case study. It was carried out in a Public Higher Education Institution, located in the interior of the state of Paraná, and brings as participants twenty-five teachers in initial training. The instuments used were questionnaires with open questions. The data were analyzed from the content analysis, divided into three categories: i) conception about visual impairment; ii) inclusive education; iii) assistive technology. The results reveal that there are gaps in relation to the teachers' knowledge about TA for the teaching and learning process for visually impaired students, paying attention to the inclusion of subjects in the undergraduate curriculum, to address the application of consistent theories and practices.
\end{abstract}

Keywords: Assitive technology. Dosvox Software. Teacher training. Visual impairment. Inclusion.

\section{CONCEPCIÓN DE PROFESORES EN FORMACIÓN INICIAL: CONOCIMIENTO SOBRE LA TECNOLOGÍA ASISTIVA DOSVOX}

Resumen: El uso de la Tecnología Asistiva (TA) ha aumentado la autonomía y ha permitido la inclusión de los estudiantes con discapacidad en los entornos educativos. Por ello, es importante que los profesores estén cualificados para utilizar estas tecnologías en las aulas. Este estudio tiene como objetivo investigar la concepción de los profesores en formación inicial de un curso de Pedagogía sobre el conocimiento de la tecnología de asistiva - software Dosvox para la educación inclusiva, para diseñar el proceso de enseñanza y aprendizaje de los estudiantes con discapacidad visual. Se utilizó un enfoque cualitativo de carácter descriptivo, teniendo como estrategia el estudio de casos. Se realizó en una Institución Pública de Enseñanza Superior, ubicada en el interior del estado de Paraná, y reúne como participantes a veinticinco profesores en formación inicial. Los instrumentos utilizados fueron cuestionarios con preguntas abiertas. Los datos se analizaron a partir del análisis de contenido, dividido en tres categorías: i) concepción sobre la discapacidad visual; ii) educación inclusiva; iii) tecnología asistivia. Los resultados revelan que existen lagunas en cuanto a los conocimientos de los profesores sobre las TA para el proceso de enseñanza y aprendizaje de los alumnos con discapacidad visual, prestando atención a la inclusión de asignaturas en el plan de estudios de grado, para abordar la aplicación de teorías y prácticas coherentes.

Palabras clave: Tecnología asistiva. Software dosvox. Formación de profesores. Discapacidad visual. Inclusión. 


\section{Introdução}

O uso de tecnologias vêm ganhando espaço no cenário educacional, com o objetivo de promover transformações em salas de aula. Frente a este cenário, são necessárias reflexões sobre o uso das Tecnologias Assistivas (TA) de forma inclusiva, propondo assim, novas percepções de modo mais condicionante, a possibilitar o acesso ao processo de ensino e aprendizagem de estudantes com deficiência. Considera-se que o acesso ao conhecimento por pessoas com deficiência é um direito, previsto em leis (BRASIL, 1994, 1996, 2001, 2008, [2016]).

Bersch (2017), considerou que a TA engloba recursos, metodologias, estratégias e práticas que possibilitam aos estudantes com deficiência maior autonomia, qualidade de vida e inclusão social, a organizar de acordo com as necessidades peculiares de cada estudante.

Com base neste contexto, este estudo utilizou a TA - Software Dosvox, utilizada como recurso de acesso ao conhecimento por pessoas com deficiência visual. Esta tecnologia é considerada como um sistema de programação que se comunica com os usuários através da síntese de voz, viabilizando o uso de computadores para estas pessoas, posibilitando-Ihes maior acessibilidade tecnológica (BORGES, 2009).

A TA - Software Dosvox constitui em seu sistema, um leitor de tela que utiliza de sintetizadores de voz que permite a leitura e as informações na tela do computador pelo usuário. Está disponível para pessoas com deficiência visual e outras pessoas que tenham interesse em utilizá-la. Esta tecnologia, tem como intuito permitir o acesso aos contextos digitais, informacionais, sociais e de apoio pedagógico nos espaços escolares. O programa é distribuído nas versões para Windows (BORGES, 2009; BORGES; MENDES, 2018; SONZA; SANTAROSA, 2003).

O Software Dosvox está em constante evolução, sendo desenvolvido desde o ano de 1993 pela Universidade Federal do Rio de Janeiro - UFRJ. Estudos apresentam a importância de fazer uso deste software em salas de aula, a pensar no desenvolvimento e no processo de ensino e aprendizagem de estudantes com deficiência visual (ALVES FILHO, 2009; BORGES, 2009; CANEJO, 2016; DIAS; FRANÇA; BORGES, 2014; MAZZILLO, 2010). 
Sonza e Santarosa (2003) consideram este software como uma fonte de acessibilidade para pessoas com deficiência visual. As autoras destacam que o sistema do Software faz uso de uma tecnologia totalmente nacional (Brasileira), sendo o primeiro sistema comercial a sintetizar vocalmente textos genéricos para a língua portuguesa, facilitando a acessibilidade destas pessoas ao uso do computador.

Alves Filho (2009) salienta em seus estudos que, apesar de o Software Dosvox possibilitar a acessibilidade para as pessoas com deficiência visual ao conhecimento, existe a necessidade de os professores qualificarem-se sobre a funcionalidade e uso do sistema de programação. Isto porque, a qualidade de ensino é essencial para o uso da tecnologia inclusiva em salas de aula durante o processo de ensino e aprendizagem à estudantes com deficiência visual, principalmente ao que concerne o processo de iniciação tecnológica para a aprendizagem desses estudantes, a qual, tem início nos primeiros anos do ensino fundamental.

Em relação à formação de professores, Prais e Rosa (2017) evidenciam que a temática tem gerado muitas discussões frente à necessidade emergente para se efetivar a educação inclusiva de pessoas com deficiênicia em salas de aula. Os autores apontam carências na formação pedagógica dos professores.

A perspectiva da educação inclusiva está diretamente relacionada com a formação dos professores, pois a formação infere nas políticas inclusivas e sua consolidação nos âmbitos educacionais de ensino. Portanto, há necessidade de ampliar a formação pedagógica dos professores em formação inicial para o contexto da educação inclusiva. Essa necessidade deve ser pensada de modo que contribua com qualidade do ensino inclusivo para todas as pessoas com deficiência no contexto atual (PRAIS; ROSA, 2017; SOUZA; MENDES, 2017).

Para prestar atendimento pedagógico com qualidade de ensino às pessoas com deficiência e/ou transtornos, as escolas de ensino regular precisam realizar flexibilizações curriculares e estratégias pedagógicas que consistem basicamente na tomada de decisão dos professores juntamente com a equipe pedagógica, buscando proporcionar uma ação educativa escolar que atenda as peculiaridades dos estudantes com deficiência, a 
possibilitar um currículo mais dinâmico, alterável e passível de ampliação que atenda a todos os estudantes, sem perda de conteúdo (BRASIL, 2003).

Assim, este estudo objetivou investigar sobre a concepção de professores em formação inicial de um curso Graduação em Pedagogia, sobre o conhecimento da Tecnologia Assistiva - Software Dosvox para o uso com a educação inclusiva.

Neste viés, justifica-se o presente trabalho fundamentado-se na inclusão de pessoas com deficiência visual no ensino regular e a necessidade de promover formação aos professores para o ensino inclusivo, a fazer uso de diferentes recursos tecnólogicos que contribuam para a inclusão de estudantes com deficiência visual em salas de aula, ao acesso e a apropriação do conhecimento.

Almeja-se contribuir com o processo de formação inicial de professores, no sentido de ampliar as concepções sobre o uso de TA em salas de aula à estudantes com deficiência visual (baixa visão/ cegueira total), assim como, disseminar o desenvolvimento de pesquisas na área de TA como recurso didático para o conhecimento.

\section{Encaminhamentos metodológicos}

Este estudo apresenta abordagem qualitativa, constituindo-se num estudo de natureza descritiva. Para Gil (2002, p. 42) este tipo de estudo normalmente é realizado por "pesquisadores sociais preocupados com a atuação prática".

A estratégia que atende aos objetivos propostos é o estudo de caso inédito, por se tratar de um estudo de investigação sobre o que/e quais são os conhecimentos dos professores em formação inicial sobre o processo inclusivo de estudantes com deficiência visual em salas de aula. Para Lüdcke e André (1986), o ponto central do estudo de caso incide sobre suas particularidades, mesmo que, posteriormente, evidenciem-se semelhanças com outros casos ou situações. O estudo de caso visa à descoberta, acontece de forma contextualizada, retratando a realidade de forma complexa e profunda.

O estudo foi desenvolvido em uma instituição Pública de Ensino Superior, localizada no interior do Estado do Paraná/Brasil e, contou com a participação de vinte e cinco professores em formação inicial, do segundo ano de um curso de Pedagogia, tendo maioridade no período da pesquisa. Os participantes aceitaram participar do mesmo, 
assinando-se o Termo de Consentimento Livre e Esclarecido - TCLE, subsidiando-se o termo ético e moralmente atribuído ao estudo, no sentido de não expor o público envolvido, e que a pesquisa é de caráter totalmente científico.

Utilizou-se de um questionário semi estruturado como instrumento para coleta de dados, composto por nove perguntas. Para a análise dos dados, as respostas obtidas foram divididas em três categorías, buscando-se investigar na Categoria 1: o conhecimento dos professores em formação inicial sobre a deficiência visual; Categoria 2:

o conhecimento dos professores frente a educação inclusiva e na Categoria 3: o conhecimento dos professores referente as Tecnologia Assistiva -TA inclusivas em salas de aula.

O Quadro 1 apresenta o questionário aplicado aos professores em formação inicial e as categorias utilizadas:

Quadro 1-Questionário aplicado aos professores em formação inicial

\begin{tabular}{|l|l|l|}
\hline $\begin{array}{l}\text { Categoria 1: conhecimento } \\
\text { sobre a deficiência visual }\end{array}$ & $\begin{array}{l}\text { Categoria 2: conhecimento } \\
\text { sobre a educação inclusiva }\end{array}$ & $\begin{array}{l}\text { Categoria 3: conhecimento } \\
\text { sobre a tecnologia assistiva }\end{array}$ \\
\hline $\begin{array}{l}\text { 1. Você sabe o que é a } \\
\text { deficiência visual? Descreva. }\end{array}$ & $\begin{array}{l}\text { 1. Qual é a diferença entre } \\
\text { educação inclusiva educação } \\
\text { especial? }\end{array}$ & $\begin{array}{l}\text { 1. Você tem conhecimento } \\
\text { sobre as tecnologias assistivas } \\
\text { utilizadas no processo de } \\
\text { ensino e aprendizagem de } \\
\text { pessoas com deficiência } \\
\text { visual? Quais? }\end{array}$ \\
\hline $\begin{array}{l}\text { 2. Você conhece pessoas com } \\
\text { deficiência visual? Já conviveu } \\
\text { com elas? Descreva. }\end{array}$ & $\begin{array}{l}\text { 2. Você tem experiência em } \\
\text { salas de aula com pessoas com } \\
\text { deficiência visual? Descreva. }\end{array}$ & $\begin{array}{l}\text { 2. Você já teve contato direto } \\
\text { com alguma ferramenta com } \\
\text { sintetizadores de voz? Quais? } \\
\text { Como foi a sua experiência? } \\
\text { Descreva. }\end{array}$ \\
\hline $\begin{array}{l}\text { 3. Qual seria sua atitude como } \\
\text { professor (a) se tivesse um } \\
\text { aluno (a) com deficiência } \\
\text { visual em sala de aula? De que } \\
\text { forma trabalharia com esse } \\
\text { aluno (a)? }\end{array}$ & $\begin{array}{l}\text { 3. Quais recursos didáticos e } \\
\text { metodológicos utilizaria em de aula se tivesse algum } \\
\text { aluno (a) com deficiência } \\
\text { visual? }\end{array}$ & $\begin{array}{l}\text { 3. Você já ouviu falar na } \\
\text { ferramenta Dosvox? O que é? } \\
\text { Sabervemo funciona? Pra que } \\
\text { servenlique. }\end{array}$ \\
\hline
\end{tabular}

Fonte: Acervo das autoras (2019). 
Os dados deste estudo foram contextualizados e refletidos por meio da análise de conversação. Segundo Flick (2009), o foco das análises concentra-se no contexto das informações e suas interpretações. O objetivo desta forma de análise foi de determinar os princípios e mecanismos presentes nas ações e nas reações que as ações causam.

\section{Resultados e discussão}

A Resolução CNE/CP no 1/2002, estabelece as Diretrizes Curriculares Nacionais para a Formação de Professores da Educação Básica, em nível superior, para os cursos de licenciatura. Define que as instituições de ensino superior devem prever, em sua organização curricular, formação docente voltada para a atenção à diversidade contemplando conhecimentos sobre as especificidades dos estudantes com necesidades educacionais especiais (CONSELHO NACIONAL DE EDUCAÇÃO, 2002). Com base nesta resolução foram aplicados questionários aos professores em formação inicial com o intento de investigar seus conhecimentos no processo inclusivo de estudantes com deficiência visual no uso da tecnologia assistiva.

Assim, a análise dos dados foi estruturada por meio do instrumento utilizado no estudo. O questionário aplicado aos professores em formação inicial de um curso de Pedagogia, revelou que estes professores apresentam carências relacionadas aos conhecimentos sobre a deficiência visual; educação inclusiva e a TA - Software Dosvox. A seguir são apresentadas as discussões fundamentadas nas três categorías elaboradas para as análises.

\section{Categoria 1 - conhecimento sobre a deficiência visual}

De modo geral, os professores participantes do estudo conceituaram a deficiência visual da seguinte forma:

[...] Deficiência visual é a falta de visão, ou seja, pessoas com essa deficiência não conseguem enxegar (PROFESSOR EM FORMAÇÃO 
INICIAL, 2019).

[...] Deficiência visual é a pessoa impossibilitada de enxergar (PROFESSOR EM FORMAÇÃO INICIAL, 2019).

[...] Deficiência visual é quando a pessoa sofreu alguma coisa e ficou cega (PROFESSOR EM FORMAÇÃO INICIAL, 2019).

[...] Deficiência visual refere-se a condição em que a pessoa tem algum grau de dificuldade para enxergar (PROFESSOR EM FORMAÇÃO INICIAL, 2019).

[...] Deficiência visual é quando a pessoa tem algum problema, não consegue ver, pode ser desde o nascimento ou ao longo da vida (PROFESSOR EM FORMAÇÃO INICIAL, 2019).

Diante das respostas apresentadas, considera-se que o conceito sobre a deficiência visual, vai além da concepção dos professores em formação inicial. Suas respostas aproximam-se ao senso comum. Assim, fundamentando-se em tal carência de conhecimentos específicos e científicos sobre a deficiência visual, torna-se indispensável esclarecer neste estudo sobre a temática.

Deste modo, recorreu-se a Otaiano et al. (2019), os autores apresentam algumas definições categorizando a deficiência visual de acordo com a Classificação Internacional de Doenças CID-10, como: deficiência visual leve ou sem deficiência; deficiência visual moderada; deficiência visual severa e a cegueira com ou sem percepção de luz, a considerar a acuidade visual pela distância.

Otaiano et al. (2019, p. 14) apresentam em seus estudos que a primeira estimativa global sobre deficiência visual foi datada de 1972, em um levantamento feito pela Organização Mundial da Saúde - OMS, e indicou, na época que existe cerca de "[...] 10 a 
15 milhões de pessoas cegas no mundo, as estimativas de crescimento preveem que a população mundial chegue a 8,6 bilhões até 2030 , sendo a maior parte desse crescimento populacional prevista para os países em desenvolvimento".

A definição legal sobre a acuidade visual para Malta et al. (2016, p. 3255) se constitui "[...] igual ou menor que 0,05 no melhor olho, com a melhor correção óptica; a baixa visão, que significa acuidade visual entre 0,3 e 0,05 no melhor olho, com a melhor correção óptica". Os autores evidenciam em seus estudos sobre a prevalência dentre várias deficiências, a mostrar que 3,6\% da população brasileira possui deficiência visual, o que equivale a aproximadamente $7,2 \%$ da população considerando homens e mulheres, com tendência a acrescer com o passar dos anos, devido ao aumento de doenças que podem ocasionar a perda da visão, como, por exemplo, a doença retinopatia diabética, cataratas, glaucoma, dentre outras.

Tendo em vista a demanda significativa de pessoas com deficiência visual, assim como de estudantes com essa deficiência em escolas de rede regular de ensino, considera-se fundamental que o profesor, em especial, em formação inicial adquira conhecimentos sobre os conceitos, as definições e peculiaridades na área da deficiência visual, pois tais conhecimentos estão interligados diretamente com o processo de ensino e aprendizagem em salas de aula.

\section{Categoria 2 - conhecimento sobre a educação inclusiva}

A educação inclusiva tem sido discutida no Brasil, devido ao aumento significativo de estudantes em salas de aula com algum tipo de deficiência. Para Prais e Rosa (2017) a maior preocupação é com a formação dos professores. Nesta perspectiva, o questionário aplicado aos professores em formação inicial buscou compreender, para além da deficiência visual, no sentido de investigar como estes professores compreendem a Educação Especial e a Educação Inclusiva e os recursos didáticos e metodológicos utilizados em salas de aula inclusivas.

Constatou-se que, os professores em formação inicial apresentaram um conhecimento efêmero sobre a temática. Para melhor respaldo, destacam-se alguns trechos das respostas dos mesmos: 
[...] a educação inclusiva é fazer com que todos os alunos façam as mesmas atividades (PROFESSOR EM FORMAÇÃO INICIAL, 2019).

[...] a educação inclusiva busca inserir os alunos no ensino regular (PROFESSOR EM FORMAÇÃO INICIAL, 2019).

[...] a educação inclusiva apenas coloca esses alunos em sala de aula (PROFESSOR EM FORMAÇÃO INICIAL, 2019).

[...] Não tenho ideia o que é educação inclusiva (PROFESSOR EM FORMAÇÃO INICIAL, 2019).

[...] não tenho certeza, mas acredito que a educação inclusiva você insere o aluno que tem dificuldades em uma sala de aula (PROFESSOR EM FORMAÇÃO INICIAL, 2019).

[...] a educação especial é trabalhar separadamente sem a inclusão da pessoa com deficiência (PROFESSOR EM FORMAÇÃO INICIAL, 2019).

Diante das respostas acima apresentadas, considerou-se que eles carecem de ações pedagógicas que possam direcioná-los para uma concepção mais fundamentada sobre a Educação Especial e a Educação Inclusiva. Percebe-se diversas contradições e fragmentações em suas respostas. Desse modo, para melhor clarificar tal concepção, fundamentou-se com base na Política Nacional de Educação Especial na Perspectiva da Educação Inclusiva (BRASIL, 2008, p. 10), a qual evidencia que:

[...] a educação especial é uma modalidade de ensino que perpassa 
todos os níveis, etapas e modalidades, realiza o atendimento educacional especializado, disponibiliza os recursos e serviços e orienta quanto a sua utilização no processo de ensino e aprendizagem nas turmas comuns do ensino regular [...] acompanhado por meio de instrumentos que possibilitem monitoramento e avaliação da oferta realizada nas escolas da rede pública e nos centros de atendimento educacional especializados públicos ou conveniados.

Brasil (2008, p. 11) salienta-se que o atendimento educacional especializado na Educação Especial no ensino superior, as ações pedagógicas devem promover o acesso, a permanência e a participação dos estudantes com quaisquer tipos de deficiências. Essas ações inclusivas envolvem:

[...] planejamento e a organização de recursos e serviços para a promoção da acessibilidade arquitetônica, nas comunicações, nos sistemas de informação, nos materiais didáticos e pedagógicos, que devem ser disponibilizados nos processos seletivos e no desenvolvimento de todas as atividades que envolvam o ensino, a pesquisa e a extensão.

Nesse viés, a Educação Inclusiva fundamenta-se na concepção de direitos humanos, que conjuga igualdade e diferença como valores indissociáveis, e que avança em relação à ideia de eqüidade (BRASIL, 2008). O processo da educação inclusiva brasileira está muito engajado à formação de professores, a considerar que as ações tem como intento a transfomação deste cenário.

\section{Categoria 3 - conhecimento sobre a tecnologia assistiva}

A TA é uma terminologia utilizada para identificar todo o conjunto de recursos e serviços que contribuem e ampliam as habilidades funcionais de pessoas com deficiência, posibilitando a elas independência e inclusão digital. Especificamente para as pessoas com deficiência visual, são disponibilizados recursos como, por exemplo, lupas e lentes, braille para equipamentos com síntese de voz, grandes telas de impressão, sistema de TV com aumento para leitura de documentos, equipamentos de entrada e saída (síntese de voz, braille), teclados modificados ou alternativos se necessário, acionadores, softwares 
especiais (de reconhecimento de voz) que possibilitam as pessoas com deficiência a usar o computador (BRASIL, 2009).

Ao que se refere à TA, verificou-se nas respostas apresentadas pelos sujeitos da pesquisa, o desconhecimento sobre o uso da Tecnologia Assistiva desenvolvida para estudantes com deficiência visual. Dos vinte e cinco participantes que responderam os questionários, um respondeu que tinha conhecimento sobre a área, sem maiores interferências.

Considera-se a indispensabilidade de que o profesor em formação inicial, adquira conhecimentos sobre o que constitui a Tecnologia Assistiva em salas de aula e como usála em prol do ensino e aprendizagem dos estudantes. Borges e Mendes (2018, p. 484) destacam que a TA é uma "[...] área de conhecimento interdisciplinar que compreende produtos, recursos, metodologias, estratégias, práticas e serviços que objetivam promover a funcionalidade para pessoas com deficiência". As autoras enfatizam ainda a importância das ações pedagógicas advindas dos professores, pois essas ações implicam diretamente no processo inclusivo. Porém, essas ações requerem maior formação dos professores, a fim de atender essas expectativas sobre o uso de TA como recursos inclusivos em salas de aula.

Especificamente ao que se refere ao Software Dosvox, obteve-se algumas respostas transcritas a seguir:

[...] nunca ouvi falar nessa ferramenta (PROFESSOR EM FORMAÇÃO INICIAL, 2019).

[...] Não sei o que é isso (PROFESSOR EM FORMAÇÃO INICIAL, 2019).

[...] Não conheço esse sistema (PROFESSOR EM FORMAÇÃO INICIAL, 2019).

[...] Não tenho conhecimento sobre esta ferramenta (PROFESSOR EM FORMAÇÃO INICIAL, 2019).

[...] Não sei bem o que é, mas acredito que seja uma ferramenta 
que lê o que está escrito nos computadores para a pessoa cega (PROFESSOR EM FORMAÇÃO INICIAL, 2019).

De modo geral, os profesores em formação inicial esclarecem em suas respostas não obter conhecimento sobre a TA e, tampouco sobre o Dosvox como recurso didático ao processo de ensino e aprendizagem de pessoas com deficiência visual.

Tais concepções, quanto ao conhecimento das TA inclusivas, podem ser sanadas por estes professores. Após a constatação explicita pelos professores sobre suas dificuldades e dúvidas referentes a deficiência visual, educação inclusiva e a Tecnologia Assistiva, foi desenvolvido um plano de ação, o qual abordou intervenções práticas no uso do computador para mediar aos professores em formação inicial conhecimentos sobre tais categorias discutidas neste estudo, em específico sobre a TA - Software Dosvox.

O diferencial do estudo é que tal intervenção, para com os profesores em formacao inicial, imersos em curso de Graduacao em Pedagogia, segunda série, foi aplicado por um professor cego responsável pelo ensino de TA para pessoas com deficiência visual no municipio, e, o intuito de tais práticas foi iniciar a desmistificação sobre o processo de ensino e aprendizagem na Educação Inclusiva. Os resultados de tais estudos práticos serão futuramente apresentados em outros estudos, pois não há como esgotar um conteúdo tão vasto e primordial para discussões da academia, principalmente para a formação docente na sociedade contemporânea.

\section{Considerações finais}

O estudo objetivou-se investigar a concepção de professores em formação inicial de um curso de Graduação em Pedagogia sobre o conhecimento da Tecnologia Assistiva Software Dosvox para Educação Inclusiva, no sentido de possibilitar reflexões sobre o processo de ensino e de aprendizagem de estudantes com deficiencia visual.

Os resultados revelaram inicialmente que, existem lacunas na formação inicial a respeito da inclusão de pessoas com deficiência visual, bem como ao aspecto sobre o seu ensino, assim como pouco conhecimento incipiente sobre o uso da TA no processo inclusivo em salas de aula. As reflexões e discussões ocorridas posibilitam análises para ações na formação inicial de professores, principalmente ao que tange o processo de educação inclusiva tecnológica em salas de aula, tendo em vista o aumento de estudantes 
com deficiência visual matriculados na rede regular de ensino.

Haja vista, tal carência em relação as categorias do conhecimento abordadas neste estudo a: i) concepção sobre deficiência visual; ii) educação inclusiva; iii) tecnologia assistiva, considera-se de suma importância a inclusão de disciplinas que abordem questões referentes ao processo de ensino e aprendizagem para pessoas com deficiência no currículo das licenciaturas, a considerar a aplicação de teorias e práticas consistentes ao ensino, a proporcionar aos professores em formação inicial, o acesso ao conhecimento relacionado às características e definições de pessoas com deficiência, transtornos globais do desenvolvimento, altas habilidades/superdotação (BRASIL, 2008). Assim como, a apresentar recursos de TA que podem ser utilizados, a promover a aprendizagem e o desenvolvimento, projetando problematizações e reflexões para quando estes professores estiverem em uma sala de aula inclusiva, com intento de que estes professores compreendam como e quais são as posibilidades para o processo de ensino e aprendizagem para o público alvo da Educação Especial.

Contudo, o presente estudo torna-se instigante, haja vista a necessidade de reflexões sobre o assunto de TA e Educação Inclusiva, bem como as possiblidades das quais estas abrangem dentro do processo de construção do conhecimento. Assim, não há como esgotar o assunto, tendo a necessidade de contínuos experimentos e contextos, para que a academia tenha cada vez mais subsídios teórico-práticos para que o acesso à educação seja direcionado aos que nela priorizam, em especial, para com a formação docente inicial.

\section{Referências}

ALVES FILHO, Teófilo. Tecnologia assistiva para uma escola inclusiva: apropriação, demandas e perspectivas. 2009. Tese (Doutorado) - Universidade Federal da Bahia, Bahia, 2009. Disponível em:

https://repositorio.ufba.br/ri/bitstream/ri/10563/1/Tese\%20Teofilo\%20Galvao.pdf. Acesso em: 12 jun. 2020. 
BERSCH, Rita. Introdução à tecnologia assistiva. Porto Alegre: Assistiva: tecnologia e educação, 2017. Disponível em:

https://www.assistiva.com.br/Introducao_Tecnologia_Assistiva.pdf.Acesso em: 5 fev. 2020.

BORGES, Antonio. Do Braille ao Dosvox: diferenças nas vidas dos cegos brasileiros. 2009. Tese (Doutorado) - Universidade Federal do Rio de Janeiro, Rio de Janeiro, 2009. Disponível em: http://intervox.nce.ufrj.br/dosvox/textos/tese_antonio_borges.pdf. Acesso em: 21 mar. 2020.

BORGES, Wanessa Ferreira; MENDES, Enicéia Gonçalves. Usabilidade de aplicativos de tecnologia assistiva por pessoas com baixa visão. Rev. Bras. Ed. Esp., Marília, v. 24, n. 4, p. 483-500, out./dez. 2018. Disponível em:

http://www.scielo.br/scielo.php?script=sci_abstract\&pid=S141365382018000400483\&lng $=e n \& n r m=i s o \& t$ tng=pt. Acesso em: 20 abr. 2020.

BRASIL. Saberes e Práticas da Inclusão. Estratégias para a educação de alunos com necessidades educacionais especiais. Brasília: MEC, [2003].Disponível em: http://www.dominiopublico.gov.br/download/texto/me000428.pdf. Acesso em: 10 mar. 2020.

BRASIL. Subsecretaria Nacional de Promoção dos Direitos da Pessoa com Deficiência. Comitê de Ajudas Técnicas. Tecnologia Assistiva. Brasília. CORDE, [2009]. Disponível em: https://institutoitard.com.br/produto/livro-tecnologia-assistiva-do-comite-de-ajudastecnicas/. Acesso em set. 2020.

BRASIL. [Constituição (1988)]. Constituição da República Federativa do Brasil de 1988. Brasília: Presidência da República, [2016]. Disponível em: http://www.planalto.gov.br/ccivil_03/constituicao/constituicao.htm. Acesso em: 12 jun. 2020.

BRASIL. Lei no 9.394, de 20 de Dezembro de 1996. Estabelece as diretrizes e bases da educação nacional. Brasília: Presidência da República, [1996]. Disponível em: http://www.planalto.gov.br/ccivil_03/leis/19394.htm. Acesso em: 12 jun. 2020.

BRASIL. Ministério da Educação. Declaração de Salamanca e princípios, políticas e práticas na área das necessidades educativas especiais. Brasília: MEC, [1994]. Disponível em: http://portal.mec.gov.br/seesp/arquivos/txt/salamanca.txt. Acesso em: 15 mar. 2020.

BRASIL. Ministério da Educação. Diretrizes nacionais para educação especial na educação básica. Brasília: Secretaria de Educação Especial: MEC: SEESP, [2001]. Disponível em: http://portal.mec.gov.br/seesp/arquivos/pdf/diretrizes.pdf. Acesso em: 13 set. 2020.

BRASIL. Ministério da Educação. Política nacional de educação especial na perspectiva da educação inclusiva. Brasília: [MEC], [2008]. Disponível em:

http://portal.mec.gov.br/arquivos/pdf/politicaeducespecial.pdf. Acesso em: 12 jan. 2020.

CANEJO, Elizabeth. Dosvox: rompendo barreiras da comunicação. Journal of Research in Special Educational Needs, London, v. 16, n. s1, p. 399-401. 2016. Disponível em: 
https://onlinelibrary.wiley.com/doi/epdf/10.1111/1471-3802.12299. Acesso em: 18 set. 2020.

CONSELHO NACIONAL DE EDUCAÇÃO. Resolução CNE/CP 1, de 18 de Fevereiro de 2002. Institui Diretrizes Curriculares Nacionais para a Formação de Professores da Educação Básica, em nível superior, curso de licenciatura, de graduação plena. Brasília: CNE, 2002. Disponível em:

http://portal.mec.gov.br/index.php?option=com_docman\&view=download\&alias=159261 -rcp001-02\&category_slug=outubro-2020-pdf\&Itemid=30192. Acesso em: 12 ago. 2020.

DIAS, Angélica; FRANÇA, Juliana; BORGES, Antônio. Jogavox: uma abordagem de aprendizagem colaborativa com pessoas deficientes visuais. Nuevas Ideas em Informática Educativa TISE, [Santiago], v. 10, p. 358-366, 2014. Disponível em: http://www.tise.cl/volumen10/TISE2014/tise2014_submission_324.pdf. Acesso em: 17 set. 2020.

FLICK, Uwe. Introdução à pesquisa qualitativa. 3. ed. Porto Alegre: Artmed, 2009.

GIL, Antonio Carlos. Como elaborar projetos de pesquisa. 4. ed. São Paulo: Editora Atlas, 2002.

LÜDKE, Menga; ANDRÉ, Marli. Pesquisa em educação: abordagens qualitativas. São Paulo: EPU, 1986.

MALTA, et al. Prevalência autorreferida de deficiência no Brasil, segundo a Pesquisa Nacional de Saúde, 2013. Revista Ciência \& Saúde Coletiva. Rio de Janeiro, v. 21, n. 10, p. 3253-3264. [2016]. Disponível em: https://www.scielo.br/scielo.php?script=sci_arttext\&pid=S141381232016001003253. Acesso em: 21 mai. 2020.

MAZZILLO, Beatriz Ida. Dosvox o que você deseja?. Rio de Janeiro: Ed. Universidade Federal do Rio de Janeiro, 2010.

OTTAIANO, José Augusto et al. As condições de saúde ocular no Brasil. São Paulo: Conselho Brasileiro de Oftalmologia, 2019. Disponível em: http://www.cbo.com.br/novo/publicacoes/condicoes_saude_ocular_brasil2019.pdf. Acesso em: 15 jul. 2020.

PRAIS, Souza Lidiane Jacqueline; ROSA, Flor Vanderley: A Formação de professores para inclusão tratada na Revista Brasileira de Educação Especial: uma análise. Revista Educação Especial, Santa Maria, v. 30, n. 57, p. 129-144, jan/abr. 2017. Disponível em: < https://periodicos.ufsm.br/educacaoespecial/article/view/19833. Acesso em: 12 ago. 2020. 
SONZA, Andréa; SANTAROSA, Lucila. Ambientes digitais virtuais: acessibilidade aos deficientes visuais. Revista RENOTE: novas tecnologias da educação, Porto Alegre, v. 1, n. 1, p. 1-11, fev. 2003. Disponível em: http://seer.ufrgs.br/renote/article/viewFile/13637 /7715. Acesso em: 9 ago. 2019.

SOUZA, Christianne Thatiana Ramos; MENDES, Enicéia Gonçalves. Revisão sistemática das Pesquisas colaborativas em educação especial na perspectiva da inclusão escolar no Brasil. Revista Brasileira de Educação Especial, Marília, v. 23, n. 2, p. 279-292, abr./jun. 2017. Disponível em: http://www.scielo.br/pdf/rbee/v23n2/1413-6538-rbee-23020279.pdf. Acesso em: 10 ago. 2020.

Recebido em: 02 out. 2020

Aceite em: 9 dez. 2020 